\title{
6 Fazit
}

Zusammenfassend verdeutlichen die Ergebnisse dieser Studie, dass fast alle Bereiche der rechtlichen Regelungen zur Schweigepflicht auch regelmäßig Anwendung in der Praxis finden, sodass diesen im psychotherapeutischen Alltag eine große Relevanz zukommt. Gleichzeitig zeigen sich jedoch erhebliche Defizite im Kenntnisstand der Psychotherapeuten in Bezug auf diese Regelungen, was auch ein gewisses Gefühl der Überforderung mit sich bringt. Die Unsicherheit in der Anwendung in bestimmten Situationen scheint durch das Hinzuziehen vieler unterschiedlicher Hilfsmittel allerdings nicht als übermäßig kritisch wahrgenommen zu werden, eher zeigt sich dagegen eine allgemeine Belastung durch die Einhaltung der Schweigepflicht.

Obwohl es Hinweise darauf gibt, dass insbesondere eine längere Berufserfahrung zu einem verbesserten Kenntnisstand beiträgt, können Ansätze für eine verbesserte Wissensvermittlung aufgezeigt werden, die die mangelnde Erfahrung kompensieren oder Hilfestellungen ermöglichen. So sollten insbesondere verbesserte Fortbildungen und Seminare in der Ausbildung genutzt werden, um mithilfe konkreter Beispiele und Rollenspiele das Vorgehen in relevanten Anwendungsbereichen zu verdeutlichen. Darüber hinaus kann auch die Inanspruchnahme eines schnell verfügbaren Rechtsbeistandes sinnvoll sein.

Wünschenswert wäre die Sensibilisierung für die vielfältigen Berührungspunkte und die Relevanz der Schweigepflicht im psychotherapeutischen Alltag sowie die Gewinnung von Sicherheit in der Anwendung der Normen, damit die Schweigepflicht als grundlegendes Element der Therapie das Vertrauensverhältnis stärken und den Outcome verbessern kann.

"Ich finde das ein total wichtiges Thema. Man ist sich da oft gar nicht so, oder das ist so unser wichtigster Punkt, auch so ganz sensibler Punkt und ja, ich glaube schon, dass man damit, man sich zu wenig Gedanken drüber macht. [...] Aber es ist genauso wichtig, wie auch therapeutische Interventionen, sich damit auszukennen und .. sollte man sich vielleicht an seine eigene Nase fassen und sich da noch ein bisschen besser informieren." (16, Z. 273-279)

(C) Springer Fachmedien Wiesbaden GmbH, ein Teil von Springer Nature 2019

A. Cropp, Rechtliche Grundlagen der Schweigepflicht, BestMasters, https://doi.org/10.1007/978-3-658-25092-8_6 